\title{
Utilização de medicamentos e risco de interações medicamentosas em uma instituição de longa permanência para idosos do Vale do Paraíba-SP
}

\section{Use of medicines and risk of drug interactions in a long-term care facility for the elderly in Vale do Paraíba-SP}

DOI: $10.46814 / \operatorname{lajdv3n2-003~}$

Recebimento dos originais: 23/12/2021

Aceitação para publicação: 26/02/2021

\author{
Fabíola Monteiro Querido Nascimento \\ Especialista em Enfermagem do Trabalho \\ Centro Universitário Teresa D’Àvila - Unifatea, Lorena, SP \\ Av. Dr. Peixoto de Castro, 539 - Vila Celeste, Lorena - SP, 12606-580 \\ E-mail: biola_querido@hotmail.com \\ Claudia Lysia de Oliveira Araújo \\ Doutora em Enfermagem \\ Centro Universitário Teresa D’Àvila - Unifatea, Lorena, SP \\ Av. Dr. Peixoto de Castro, 539 - Vila Celeste, Lorena - SP, 12606-580 \\ E-mail: claudialysia@gmail.com \\ Natália Lemes Siqueira Aguiar de Souza \\ Especialista em Docência em Enfermagem \\ Centro Universitário Teresa D’Àvila - Unifatea, Lorena, SP \\ Av. Dr. Peixoto de Castro, 539 - Vila Celeste, Lorena - SP, 12606-580 \\ E-mail: natalialemes0@gmail.com
}

\section{RESUMO}

Estudo exploratório e retrospectivo, de abordagem quantitativa, que objetivou avaliar a polimedicação e os potenciais riscos de interações medicamentosas em idosos de uma instituição de longa permanência do Vale do Paraíba. A população foi constituída por 40 idosos institucionalizados, com predominância do sexo feminino e média de idade de 75,15 anos. Verificou-se que 38 idosos (95\%) fazia uso de algum medicamento. A média do número de medicamentos por idoso foi de 5,8 medicamentos, sendo que o evento de polifarmácia, ou seja, o uso concomitante de cinco ou mais fármacos, ocorreu em 25 (62,5\%) prontuários analisados. Foram encontradas um total de 127 interações entre medicamentos, onde $32(80 \%)$ idosos deste estudo estavam expostos às interações medicamentosas, ficando evidente a relação direta entre a polimedicação e a possível ocorrência de interações medicamentosas.

Palavras-chave: Enfermagem, Gerontologia, Instituição de Longa Permanência para Idosos.

\begin{abstract}
Exploratory and retrospective study, with a quantitative approach, which aimed to evaluate polymedication and the potential risks of drug interactions in the elderly of a long-term institution in Vale do Paraíba. The population consisted of 40 institutionalized elderly, with a predominance of females and a mean age of 75.15 years. It was found that 38 elderly people (95\%) used some medication. The average number of medications per elderly person was 5.8 medications, and the polypharmacy event, that is, the concomitant use of five or more drugs, occurred in $25(62.5 \%)$ medical
\end{abstract}


records analyzed. A total of 127 drug interactions were found, where $32(80 \%)$ elderly people in this study were exposed to drug interactions, making the direct relationship between polymedication and the possible occurrence of drug interactions evident.

Keywords: Nursing, Gerontology, Long Term Care Facility for the Elderly.

\section{INTRODUÇÃO}

Segundo Costa e Mercadante (2013), a Sociedade Brasileira de Geriatria e Gerontologia (SBGG) adotou a expressão "Instituição de Longa Permanência para Idosos" (ILPI) para designar o tipo de instituição anteriormente chamado de "Asilo". A SBGG define-a como local para atendimento integral institucional, onde o público alvo são pessoas de 60 anos ou mais, dependentes ou independentes, que são desprovidas de condições para permanecer com a família ou sozinhas em domicílio. E com o aumento da população senil, pode-se prever que, a cada ano, mais idosos vão necessitar desses serviços.

É evidente a importância de uma avaliação adequada no momento da prescrição de medicamentos para idosos e, para que isso ocorra, é necessária a atuação de uma equipe multidisciplinar, com profissionais competentes. Deste modo, é necessário cumprir a legislação, que exige a permanência de uma equipe de profissionais de saúde nestas instituições, o que permite um acompanhamento adequado e individualizado (SMANIOTO \& HADDAD, 2013).

O desenvolvimento dos medicamentos representa um grande progresso na história da ciência e contribui para a melhoria da qualidade de vida da população (MEDEIROS et al, 2011). Todavia, a possibilidade de ocorrer um agravo induzido em decorrência da utilização de fármacos, mesmo quando utilizados nas doses prescritas e com indicação terapêutica adequada, se constitui uma realidade (CIPOLLE; STRAND; MORLEY, 2006).

A Organização Mundial da Saúde (2002) estima que mais da metade de todos os medicamentos são prescritos, dispensados ou comercializados inadequadamente, e que, aproximadamente $50 \%$ de todos os pacientes não fazem o uso correto destes.

O progresso da medicina e o uso de medicamentos são fatores importantes para o aumento da longevidade, trazendo muitos benefícios aos idosos, se utilizados com cuidado e responsabilidade. Caso contrário, os fármacos podem piorar as condições de vida desta população devido às inúmeras reações adversas aos medicamentos (RAM's) que podem acometê-los. (PASSARELLI \& FILHO, 2007). 
Interações medicamentosas são respostas farmacológicas peculiares, onde os efeitos de um ou mais medicamentos são modificados pela administração simultânea ou anterior de outros, ou através da administração concorrente com alimentos (SECOLI, 2001).

A elevada prevalência de doenças crônicas não transmissíveis faz dos idosos grandes consumidores de medicamentos e, com isso, as RAM's podem ser mais severas e acentuarem-se na população senil devido às alterações nos processos de absorção, distribuição, metabolismo e excreção dos medicamentos, característicos do envelhecimento (GAUTÉRIO et al, 2012; SMANIOTO \& HADDAD, 2013).

O idoso é especialmente vulnerável às RAM's devido aos fatores que o caracterizam: presença de múltiplas doenças, uso concomitante de diversos medicamentos (polifarmácia), particularidades farmacocinéticas e farmacodinâmicas próprias do envelhecimento e o tipo de medicamento prescrito (se é adequados ou não), visto que determinados medicamentos não deveriam ser prescritos para idosos por apresentarem riscos elevados de reações adversas. (PASSARELLI \& FILHO, 2007).

Rang et al (2004) cita alguns fatores importantes como os responsáveis pela variação na resposta ao fármaco, são eles: a etnia, idade, gravidez, fatores genéticos, doenças e interações entre os fármacos. A principal razão para a idade afetar a ação do fármaco é que a eliminação deste é menos eficaz em recém-nascidos e em idosos, visto que os fármacos geralmente produzem efeitos maiores e mais prolongados nos extremos da vida. Outros fatores relacionados com a idade, como as variações na sensibilidade farmacodinâmica, são também importantes no caso de alguns medicamentos. Alguns fatores fisiológicos e patológicos que são comuns em pessoas idosas, também influenciam os efeitos dos fármacos. A composição corpórea altera com a idade, a gordura contribuindo com uma maior proporção para a massa corpórea na velhice, com consequentes alterações no volume de distribuição dos fármacos. Os idosos consomem mais fármacos do que os adultos mais jovens e, assim, o potencial para as interações entre fármacos é também aumentado.

A equipe de enfermagem realiza um papel importante no tratamento e na terapêutica do paciente, sendo a responsável legal pela administração dos medicamentos que são prescritos. (COSTA \& MERCADANTE, 2013). Por isso, é imprescindível que a equipe de enfermagem e, principalmente o enfermeiro, tenha conhecimento a respeito da farmacologia de modo que possa identificar possíveis interações entre os medicamentos, incompatibilidades farmacológicas e reações adversas precocemente, minimizando os riscos ao cliente. (SMANIOTO \& HADDAD, 2013).

A administração de medicamentos é de responsabilidade da equipe de enfermagem, e trata-se de um procedimento que exige responsabilidade e embasamento científico para ser desenvolvida de forma segura ao paciente (SMANIOTO \& HADDAD, 2013). 
O artigo 11, inciso III, alínea “a” do Decreto de Lei n” 94.406/87 determina que a administração de medicamentos é uma atribuição do auxiliar de enfermagem, juntamente com o artigo 13 que responsabiliza o enfermeiro pela supervisão, orientação e direção desta atividade (CONSELHO FEDERAL DE ENFERMAGEM, 2014).

A quantidade diversificada de medicamentos que é consumida é o principal fator de risco para as reações adversas, pois há relação direta entre a polifarmácia e a probabilidade de reações adversas ao medicamento, interações medicamentosas e medicamentos inapropriados para idosos (LUCCHETTI et al, 2010).

A polifarmácia é definida como o uso de cinco ou mais medicamentos e está associada ao aumento do risco e da gravidade de ocorrências de reações adversas aos medicamentos, de precipitar interações medicamentosas, de causar toxicidade cumulativa, de acarretar erros de medicação e até mesmo levar a morbimortalidade. Além disso, merece atenção especial, visto que medicamentos são substâncias químicas que podem interagir entre si, com nutrientes ou agentes químicos ambientais e desencadear respostas indesejadas. (SECOLI, 2001).

Smanioto e Haddad (2013) propõem algumas medidas que visam a redução da polifarmácia e, consequentemente das interações medicamentosas, como manter o registro das medicações atualizadas e revisando-o a cada consulta, identificar o nome dos medicamentos pelo nome genérico e grupo terapêutico, assegurar-se da indicação adequada, ter conhecimento dos efeitos secundários dos medicamentos, conhecer a fisiologia do envelhecimento e evitar excessos farmacológicos.

A consequência de uma interação medicamentosa pode levar ao fracasso terapêutico ou a aparição de reações adversas. Ambas as probabilidades colocam o paciente em risco e causam outros prejuízos, como a necessidade de diagnóstico e tratamento de maior complexidade, a hospitalização do paciente ou o aumento da internação hospitalar (MATOS et al, 2009).

\section{OBJETIVO}

Avaliar a polimedicação e os potenciais riscos de interações medicamentosas em idosos de uma instituição de longa permanência do Vale do Paraíba.

\section{INTERAÇÃO MEDICAMENTOSA}

Para Gustav (2005) as interações medicamentosas são alterações nos efeitos de um medicamento em razão da ingestão simultânea de outro fármaco ou do consumo de determinado alimento. Embora em alguns casos os efeitos de medicamentos combinados sejam benéficos, é mais comum que as interações medicamentosas sejam prejudiciais. 
Matos et al (2009) menciona que as interações medicamentosas referem-se à interferência de um fármaco na ação de outro ou de um alimento ou nutriente na ação de medicamentos.

As respostas desencadeadas das interações podem potencializar o efeito terapêutico, reduzir a eficácia, surgir reações adversas com diversos graus de gravidade ou ainda, não causar nenhuma alteração no efeito desejado do medicamento. Assim, a interação entre medicamentos pode ser benéfica, causar respostas contrárias não previstas no regime terapêutico ou apresentar pequeno significado clínico (SECOLI, 2001).

Para Matos et al (2009) as interações dos medicamentos podem ser classificadas como indesejáveis ou desejáveis. Estas últimas são consideradas benéficas e conhecidas como associações por reduzir os efeitos adversos, prolongar a duração do efeito, aumentar a adesão ao tratamento e incrementar a eficácia. As indesejáveis reduzem o efeito terapêutico, aumentam a ocorrência de efeitos adversos e do custo com o tratamento.

As interações se manifestam por mecanismos farmacocinéticos e farmacodinâmicos. Os mecanismos farmacocinéticos correspondem a qualquer interação ocorrida durante a absorção, distribuição, metabolização ou excreção, podendo levar a um aumento ou redução da concentração do fármaco. Os processos farmacodinâmicos estão relacionados com a resposta da droga nos receptores ou locais de atuação, sendo que a interação pode potencializar ou anular a resposta desejada. (MATOS et al, 2009).

\section{METODOLOGIA}

A pesquisa foi realizada em uma instituição de longa permanência para idosos situada no Vale do Paraíba - SP, apresentando 40 idosos em regime de internato.

Foi realizado um estudo exploratório e retrospectivo, de abordagem quantitativa, a partir dos prontuários de 40 idosos, considerando os dados apresentados no ano de 2015. Os prontuários de todos os idosos institucionalizados foram analisados nesta pesquisa, não sendo estabelecidos critérios de inclusão e exclusão.

Para a coleta dos dados foi utilizado um instrumento de coleta de dados que incluía informações sobre cada idoso, tais como idade, sexo, quantidade de filhos, religião, estado civil, escolaridade, além dos dados da farmacoterapia prescrita. Os dados foram coletados no mês de abril do ano de 2015 e posteriormente analisados.

O desenvolvimento do estudo atendeu às normas nacionais, estabelecidas na Resolução 466/2012 do Conselho Nacional de Saúde, e internacionais de ética em pesquisa envolvendo seres humanos, recebendo aprovação sob o número CAAE 43453815.1.0000.5431. 


\section{RESULTADOS E DISCUSSÃO}

Foram analisados os prontuários de 40 idosos residentes de uma instituição de longa permanência para idosos no Vale do Paraíba, com média de idade de 75,15 anos, sendo que 21 (52,5\%) eram do sexo feminino. Este dado corrobora com Lucchetti et al (2010) e Gautério et al (2012), onde também houve ascendência da população feminina. Porém, vai de encontro com os resultados obtidos por Oliveira e Novaes (2013) e Araújo (2011), em que o número de idosos do sexo masculino na instituição de longa permanência estudada foi predominante.

Dos entrevistados 28 (70\%) eram solteiros, 29 (72,5\%) não tinham filhos, 31 (77,5\%) eram católicos, 17 (42,5\%) eram analfabetos, 14 (35\%) tinham entre 60 e 69 anos de idade. 18 (45\%) idosos dormiam de 6 a 8 horas por dia (Tabela 1).

Tabela 1 - Características dos Idosos

\begin{tabular}{|c|c|c|}
\hline Variáveis & $n=40$ & $\%$ \\
\hline \multicolumn{3}{|l|}{ Sexo } \\
\hline Masculino & 21 & 52,50 \\
\hline Feminino & 19 & 47,50 \\
\hline \multicolumn{3}{|l|}{ Estado Civil } \\
\hline Solteiro & 28 & 70,00 \\
\hline Casado & 1 & 2,50 \\
\hline Viúvos & 4 & 10,00 \\
\hline Separados & 7 & 17,50 \\
\hline \multicolumn{3}{|l|}{ Número de Filhos } \\
\hline Não possuem & 29 & 72,50 \\
\hline Um & 4 & 10,00 \\
\hline Dois & 2 & 5,00 \\
\hline Três & 3 & 7,50 \\
\hline Mais de três & 2 & 5,00 \\
\hline \multicolumn{3}{|l|}{ Religião } \\
\hline Católicos & 31 & 77,50 \\
\hline Evangélicos & 2 & 5,00 \\
\hline Espírita & 1 & 2,50 \\
\hline Sem Religião & 6 & 15,00 \\
\hline \multicolumn{3}{|l|}{ Escolaridade } \\
\hline Analfabetos & 17 & 42,50 \\
\hline Ensino Básico & 1 & 2,50 \\
\hline Ensino Fundamental & 2 & 5,00 \\
\hline Ensino Médio & 20 & 50,00 \\
\hline
\end{tabular}

Horas de Sono 


\begin{tabular}{lll} 
Menos de 6h por dia & 12 & 30,00 \\
De 6 a 8h por dia & 18 & 45,00 \\
Mais de 8h por dia & 10 & 25,00 \\
\hline Idade & 14 & 35,00 \\
\hline 60 a 69 anos & 12 & 30,00 \\
70 a 79 anos & 13 & 32,50 \\
80 a 89 anos & 1 & 2,50 \\
Mais de 90 anos &
\end{tabular}

Fonte: as autoras (2015)

Foram descritas 21 patologias diferentes. A doença com maior prevalência foi hipertensão arterial, acometendo 27 (67,5\%) idosos (Tabela 2). Verificou-se que 38 (95\%) idosos faziam uso de alguma medicação. As medicações mais consumidas pelos idosos deste estudo atuavam no sistema cardiovascular (75\%), seguidas pelo sistema nervoso (62,5\%) e pelo sistema gastrointestinal (37,5\%). Os medicamentos que agem no sistema nervoso e no sistema cardiovascular predominam neste estudo, dado este que vai ao encontro dos achados de Lucchetti et al (2010), Oliveira e Novaes (2012) e Gautério et al (2012).

Tabela 2 - Patologias Encontradas

\begin{tabular}{lll}
\hline \multicolumn{4}{c}{ Patologias } & $\mathbf{n = 4 0}$ & $\mathbf{\%}$ \\
\hline Hipertensão & 27 & 67,50 \\
Diabetes & 11 & 27,50 \\
Retardo & 10 & 25,00 \\
Hiperplasia de Próstata & 8 & 20,00 \\
Depressão & 7 & 17,50 \\
\hline \multicolumn{4}{c}{ Fonte: as autoras (2015) }
\end{tabular}

Foram encontradas 68 medicações prescritas aos idosos deste estudo. A quantidade de medicação diária por idoso variou de 0 a 13 medicamentos. A média do número de medicamentos por idoso foi de 5,8 medicamentos, sendo que o evento de polifarmácia, ou seja, o uso concomitante de cinco ou mais fármacos, ocorreu em 62,5\% dos prontuários analisados (Tabela 3).

Tabela 3 - Ocorrência de Polifarmácia

\begin{tabular}{lll}
\hline Número de Medicamentos & $\mathbf{n}=\mathbf{4 0}$ & $\mathbf{\%}$ \\
\hline 5 & 5 & 12,50 \\
6 & 3 & 7,50 \\
7 & 7 & 17,50 \\
8 & 2 & 5,00 \\
9 & 2 & 5,00 \\
10 & 3 & 7,50
\end{tabular}




\begin{tabular}{lll}
11 & 1 & 2,50 \\
12 & 1 & 2,50 \\
13 & 1 & 2,50 \\
\hline
\end{tabular}

Fonte: as autoras (2015)

Foram encontradas um total de 127 interações entre medicamentos, onde $80 \%$ dos idosos deste estudo estavam expostos às interações medicamentosas (Tabela 4), ficando evidente a relação direta entre a polimedicação e a possível ocorrência de interações medicamentosas.

Tabela 4 - Ocorrências de Interações Medicamentosas

\begin{tabular}{|c|c|c|}
\hline$n=40$ & $\begin{array}{l}\text { Quantidade de } \\
\text { medicamentos em uso }\end{array}$ & Quantidades de I. M. \\
\hline 1 & 7 & 4 \\
\hline 2 & 4 & 3 \\
\hline 3 & 2 & 0 \\
\hline 4 & 7 & 7 \\
\hline 5 & 4 & 5 \\
\hline 6 & 5 & 2 \\
\hline 7 & 10 & 8 \\
\hline 8 & 11 & 8 \\
\hline 9 & 2 & 0 \\
\hline 10 & 10 & 5 \\
\hline 11 & 4 & 3 \\
\hline 12 & 7 & 4 \\
\hline 13 & 4 & 3 \\
\hline 14 & 4 & 3 \\
\hline 15 & 2 & 1 \\
\hline 16 & 6 & 3 \\
\hline 17 & 8 & 12 \\
\hline 18 & 4 & 2 \\
\hline 19 & 7 & 4 \\
\hline 20 & 2 & 0 \\
\hline 21 & 7 & 5 \\
\hline 22 & 0 & 0 \\
\hline 23 & 7 & 5 \\
\hline 24 & 4 & 3 \\
\hline 25 & 5 & 0 \\
\hline 26 & 5 & 3 \\
\hline 27 & 8 & 4 \\
\hline 28 & 6 & 4 \\
\hline 29 & 2 & 0 \\
\hline 30 & 12 & 7 \\
\hline 31 & 13 & 4 \\
\hline 32 & 5 & 1 \\
\hline
\end{tabular}




\begin{tabular}{lll}
33 & 10 & 4 \\
34 & 7 & 1 \\
35 & 9 & 3 \\
36 & 2 & 0 \\
37 & 0 & 0 \\
38 & 5 & 1 \\
39 & 9 & 4 \\
40 & 6 & 1 \\
\hline
\end{tabular}

No ano de 2008, Zamparetti, Luciano e Galato, realizaram um estudo a partir dos prontuários de 48 idosos institucionalizados. Esses idosos faziam uso diário em média de 4,8 medicamentos. Quando analisado o número de medicamentos por idoso foi verificado que 43,8\% estavam expostos a polimedicação. Os medicamentos mais utilizados foram do sistema cardiovascular. Verificaram possíveis interações medicamentosas em $17,8 \%$ dos casos.

Em uma pesquisa realizada por Fochat et al, no ano de 2012, avaliou os prontuários de 122 idosos de uma instituição de longa permanência. Constataram que $68 \%$ dos idosos eram mulheres, $55,7 \%$ possuíam 80 anos ou mais e $67,2 \%$ eram polimedicados. Houve prevalência de doenças do sistema cardiovascular (27\%) e a maior parte dos medicamentos utilizados atuavam no sistema nervoso $(38,8 \%)$. Foram encontradas 219 possíveis interações entre medicamentos.

Uma pesquisa realizada em uma ILPI caracterizada por admitir apenas residentes femininas, analisou 23 prontuários dessas idosas institucionalizadas, onde a idade média dessa população era de 61,1 anos. Dezoito residentes faziam uso de mais de seis classes farmacológicas diferentes e as mais utilizadas na instituição foram os antipsicóticos (12\%). A análise dos prontuários permitiu identificar 57 interações entre medicamentos (LEONARDI et al, 2012).

Giacomin, Lima e Chaves (2012) analisaram 33 prontuários de idosos em uma ILPI de Minas Gerais, onde 17 residentes eram do sexo masculino e 16 do sexo feminino, com concentração maior de idosos entre 60 e 69 anos de idade. Todos os moradores faziam uso de medicamentos e 95,45\% eram adeptos à polifarmácia. Dentre os prontuários avaliados, 75,7\% demonstravam a utilização de seis ou mais medicamentos por idosos. Foram encontradas 105 interações medicamentosas.

Em 2013, Smanioto e Haddad avaliaram prontuários de 203 idosos em quatro instituições de longa permanência diferentes, onde a média de idade foi de 76,4 anos e o predomínio era da população feminina. Dentre os idosos que fizeram parte da pesquisa, 97\% faziam uso de alguma medicação e 59,9\% foram submetidos à polifarmácia. Foram prescritos, no total, 197 medicamentos distintos à população estudada. Com relação às possíveis interações medicamentosas foi detectada a ocorrência de 359 eventos e $55,7 \%$ dos idosos estavam expostos. 
Em um estudo realizado por Fochat et al (2012), foram classificados alguns medicamentos como sendo potencialmente impróprios para idosos. A maioria dessas medicações também foram encontradas neste trabalho (Tabela 5).

Tabela 5 - Medicamentos potencialmente impróprios utilizados pelos idosos.

\begin{tabular}{|c|c|c|c|c|}
\hline \multirow{2}{*}{$\begin{array}{l}\text { Medicamentos } \\
\text { potencialmente } \\
\text { impróprios }\end{array}$} & \multirow{2}{*}{ Classe Terapêutica } & \multirow{2}{*}{$\begin{array}{l}\text { Gravidade } \\
\text { do uso }\end{array}$} & \multicolumn{2}{|c|}{ Idosos } \\
\hline & & & $\mathrm{n}$ & $\%$ \\
\hline Prometazina & Anti-histamínico para uso sistêmico & Alta & 2 & 5 \\
\hline Diazepan & Neuroléptico & Alta & 3 & 7,5 \\
\hline Fluoxetina & Neuroléptico & Alta & 4 & 10 \\
\hline Sulfato ferroso & Antianêmico & Baixa & 3 & 7,5 \\
\hline Nifedipina & Bloqueadores do canal de cálcio & Alta & 3 & 7,5 \\
\hline Amitriptilina & Neuroléptico & Alta & 6 & 15 \\
\hline Tioridazina & Neuroléptico & Alta & 1 & 2,5 \\
\hline Doxazosina & Anti-hipertensivo & Baixa & 7 & 17,5 \\
\hline Cimetidina & Droga para distúrbio ácido & Baixa & 2 & 5 \\
\hline
\end{tabular}

\section{CONCLUSÃO}

De modo geral, foi identificado um maior número de idosos do sexo feminino, com idade entre 60 e 69 anos. As doenças do aparelho circulatório foram as mais frequentes e a maioria dos idosos encontravam-se polimedicados. A maior parte dos medicamentos utilizados pertencia à classe do sistema cardiovascular, do sistema nervoso e do trato gastrointestinal. Constatou-se um grande número de possíveis interações medicamentosas, as quais podem colocar em risco a população estudada. Verificou-se, também, a presença de alguns medicamentos considerados impróprios para idosos, avaliados como fármacos de alta gravidade.

Esses resultados servem como alerta para profissionais da área de saúde sobre inconformidades no uso de medicamentos no âmbito dessas instituições. Evidencia-se a necessidade de uma constante revisão da terapêutica administrada aos idosos, além da promoção de medidas educativas, inclusive, voltadas para os profissionais responsáveis pela administração dos medicamentos.

Adotar ferramentas com critérios que permitam identificar possíveis interações medicamentosas de maneira fácil e precocemente pode ser uma forma de minimizar a ocorrência desses eventos.

O enfermeiro deve apropriar-se da prática de administração de medicamentos, valorizando esta atividade como uma sequência de técnicas a serem executadas e como uma atividade pautada em conhecimentos a respeito da terapêutica medicamentosa, anatomia e fisiologia do envelhecimento 
visando um atendimento de qualidade à população idosa, não banalizando o procedimento de administração de medicamentos.

Ainda são escassos estudos que demonstram as reações adversas ao uso de medicamentos em idosos institucionalizados, de uma maneira que se possa pensar nos fatores de risco e promover meios para uma ação eficaz, antes de instalar a polifarmácia. Deste modo, são necessários estudos que objetivam identificar eventuais erros e contradições da farmacoterapia prescrita aos idosos, para que sejam modificados de modo que a prescrição do medicamento propicie à população idosa uma melhor qualidade de vida. 


\section{REFERÊNCIAS}

ARAUJO, C. L. Conhecimento de idosos sobre o uso de medicamentos e interação medicamentosa. Em: RBCEH, v.8, n.2. Passo Fundo: maio/ago. 2011, p. 188-195.

BRASIL. Resolução no 466 de 12 de dezembro de 2012. Brasília. Conselho Nacional de Saúde. Dispõe sobre ética em pesquisas envolvendo seres humanos. Brasília: Conselho Nacional de Saúde; 2012. Disponível em: http://conselho.saude.gov.br/resolucoes/2012/Reso466.pdf. Acesso em maio de 2015.

CIPOLLE, R. J.; STRAND, L. M.; MORLEY, P. C. O exercício do cuidado farmacêutico. Brasília: Conselho Federal de Farmácia; 2006.

CONSELHO FEDERAL DE ENFERMAGEM. Decreto de Lei no 94.406/87. Disponível em: <http://www.cofen.gov.br/decreto-n-9440687_4173.html>. Acesso em: outubro de 2014.

COSTA, M. C. N. S.; MERCADANTE, E. F. O idoso residente em ILPI (Instituição de Longa Permanência do Idoso) e o que isso representa para o sujeito idoso. Revista Kairós Gerontologia, v.16, n.2, p.209-222, março 2013.

FOCHAT, R. C.; HORSTH, R. B. O.; SETTE, M. S.; RAPOSO, N. R. B.; CHICOUREL, E. L. Perfil de utilização de medicamentos por idosos frágeis institucionalizados na Zona da Mata Mineira, Brasil. Em: Rev Ciênc Farm Básica Apl, v.33, n.3, p.447-454, 2012.

GAUTÉRIO, D. P.; SANTOS, S. S. C.; PELZER, M. T.; BARROS, E. J.; BAUMGARTEN, L. Caracterização dos idosos usuários de medicação residentes em instituição de longa permanência. Rev Esc Enferm USP, São Paulo, v.46, n.6, p.1394-1399, dez 2012.

GIACOMIN, M. S.; LIMA, A. T. F.; CHAVES, A. C. P. Perfil da farmacoterapia de idosos institucionalizados de uma cidade no Vale do Aço - Minas Gerais. Em: Farmácia \& Ciência, v.3, p.0119, abr/jul. 2012.

GUSTAV, S. Farmacologia na prática clínica da área de saúde: interação medicamentosa. São Paulo: Fundamento Educacional, 2005.

LEONARDI, C.; CARPES, A. D.; BACKES, D. S.; COSTENARO, R. G. S. Interações medicamentosas potenciais em idosas institucionalizadas. Em: Disciplinarum scientia, v. 13, n. 2. Santa Maria: 2012, p. 181-189.

LUCCHETTI, G.; GRANERO, A. L.; PIRES, S. L.; GORZONI, M. L. Fatores associados à polifarmácia em idosos institucionalizados. Rev Bras Geriatr Gerontol, Rio de Janeiro, v.13, n.1, p.51$58,2010$.

MATOS, V. T. G.; VASCONCELOS, E. F.; AMARAL, M. S.; TOFFOLI-KADRI, M. C. Avaliação das Interações Medicamentosas em Prescrições Hospitalares de Pacientes Sob Uso de AntiHipertensivos. Em: Lat. Am. J. Pharm, v.28, n. 4. 2009, p.501-6.

MEDEIROS, E. F. F.; MORAES, C. F.; KARNIKOWSKI, M.; NÓBREGA, O. T.; KARNIKOWSKI, M. G. O. Intervenção interdisciplinar enquanto estratégia para o Uso Racional de Medicamentos em idosos. Ciência \& Saúde Coletiva, v.16, n.7, p.3139-3149, 2011. 
OLIVEIRA, M. P. F.; NOVAES, M. R. C. G. Uso de medicamentos por idosos de instituições de longa permanência, Brasília-DF, Brasil. Em: Rev Bras Enferm, v.65, n.5. Brasília set/out 2012, p.737-44.

ORGANIZAÇÃO MUNDIAL DA SAÚDE. Promoting rational use of medicines: core components. WHO Policy Perspectives on Medicines, $\mathrm{n}^{\circ}$ 5. Geneva: WHO; 2002.

PASSARELLI, M. C. G.; FILHO, W. J. Reações adversas a medicamentos em idosos: como prevêlas? Einstein, São Paulo, v.5, n.3, p.246-251, 2007.

RANG, H. P.; DALE, M. M.; RITTER, J. M.; MOORE, P. K. Farmacologia.Tadutores: Patricia Lydie Voeux, Antonio José Magalhães da Silva Moreira. 5a edição. Rio de Janeiro: Elsevier, 2004.

SECOLI, S. R. Interações medicamentosas: fundamentos para a prática clínica da enfermagem. Em: Revista Escola de Enfermagem USP, v.35, n.1. São Paulo: mar. 2001, p. 28-34.

SMANIOTO, F. N.; HADDAD, M. C. L. Avaliação da farmacoterapia prescrita a idosos institucionalizados. Rev Bras Enferm, Brasília, v.66, n.4, p.523-527, jul/ago. 2013.

ZAMPARETTI, F.; LUCIANO, L.; GALATO, D. Utilização de Medicamentos em uma Instituição de Longa Permanência para Idosos do Sul de Santa Catarina - Brasil. Em: Lat. Am. J. Pharm. v.27, n.4, p.553-59, 2008. 